\title{
NUMERICAL ANALYSIS OF EDDY CURRENT FIELD IN LAMINATED MEDIA
}

\author{
Miklós KUCZMANN \\ Department of Automation \\ Faculty of Mechanical Engineering, Informatics and Electrical Engineering \\ Széchenyi István University, Egyetem tér 1, H-9026 Győr, Hungary \\ e-mail: kuczmann@sze.hu
}

Received 30 October 2017; accepted 5 January 2018

\begin{abstract}
A comprehensive analysis of the finite element method based lamination modeling has been performed and the results are presented in this paper. The simulations are made in two subsequent steps. In the first step, the approximate magnetic field distribution inside the material with linear characteristics is determined assuming a bulk material having anisotropic conductivity and laminates are not taken into account. In the second step, the eddy current field inside the individual laminates is modeled. The boundary conditions of any individual sheets are obtained from the bulk model. The paper presents the advantages and the drawbacks of the applicable potential formulations. Results are compared with the quasi-static electromagnetic field obtained from a reference solution taking account of each laminate.
\end{abstract}

Keywords: Lamination modeling, Eddy current field, Finite element method

\section{Introduction}

Lamination plays an important role in electrical machines. Stator and rotor of rotating machines as well as transformer cores and devices in power electronics are made of laminated sheets to decrease the losses caused by eddy currents induced inside the steel laminates. The Thesis [1] has reported that the geometry and the material parameters pose a nonlinear three dimensional electromagnetic field problem.

A key aspect of eddy current simulations is the prediction of losses due to eddy currents. However, in the case of laminated media simulations, this task is computationally intensive, because the lamination thickness is usually much smaller than the other dimensions of the problem. Three dimensional computations of real-life 
problems taking each of the laminations into consideration leads to an extremely high computational task that cannot be realized today. This results in a large number of finite elements, i.e. a large number of unknowns.

There are possibilities to decrease the computational costs. A detailed review of the relevant literature can be found in [1], [2], here a short summary is presented. The most common way is assuming non-laminated bulk material with anisotropic conductivity [3]. The magnetic field approximated by the bulk model is acceptable, although the eddy currents and the loss are not correct [4]-[6]. A single component electric vector potential is proposed in [7] to consider the laminar nature of eddy currents. Aside from edge effects, one dimensional and two dimensional models can be applied to predict losses due to field parallel to the laminations. These methods are computationally not intensive, but fail to describe the three dimensional eddy current distributions. A detailed study about these models focusing on vector hysteresis in rotating machines can be found in [8]. Three dimensional models can take the geometry into account, and the losses caused by parallel and normal components of the field can be computed [2].

This paper is focusing on linear three dimensional problems. Although previous literature has examined the present problem [2], [5], [6], a comprehensive analysis of the finite element formulations has been performed to investigate them.

The aim of this paper is to compare the potential formulations in the frame of a comprehensive analysis to choose the most applicable one. The three dimensional eddy current distributions can be simulated in the laminations without to resort to a large finite element model representing each laminate. All the methods have two steps. In the first step, a so called bulk model is analyzed. The true geometry of laminations is not taken into account, but the conductivity is anisotropic, having zero or very low value in the direction normal to the sheets. The solution of the bulk model considers the magnetic stray field and the large laminar eddy current loops due to the magnetic stray field [3]. It can be checked by a reference model.

For the reference, the ungauged $\boldsymbol{T}, \Phi-\Phi$-formulation is used. For the bulk model, here, the $\boldsymbol{T}, \Phi-\Phi$, the $\boldsymbol{A}, V-\boldsymbol{A}$ and the $\boldsymbol{A}, \boldsymbol{T}-\boldsymbol{A}$-formulations are used, but many other formulations can be applied [9]-[11].

In the second step, all the laminates with the true geometry are analyzed. The boundary conditions of this model are obtained from the bulk model and the $T, \Phi$, the $\boldsymbol{A}, V$, and the $\boldsymbol{A}, \boldsymbol{T}$-formulations are used to represent the eddy current field. The eddy current field within the laminations can be calculated in a parallel way, because the models are independent from each other. This advantageous technique results in a fast and efficient calculation of eddy current losses.

For the illustration (see Fig. 1), a $10 \mathrm{~mm} \times 10 \mathrm{~mm} \times 10 \mathrm{~mm}$ cube built up from ten laminates $\left(\mu_{r}=1000\right.$ is the value of relative permeability, the conductivity is $\left.\sigma=5.875 \cdot 10^{6} \mathrm{~S} / \mathrm{m}\right)$ is immersed in the magnetic field of a cylindrical coil supplied by sinusoidal current (peak value of the current is $4800 \mathrm{~A}$-turn, $f=50 \mathrm{~Hz}$, the inner and outer radii and the length of the coil are $7.1 \mathrm{~mm}, 11 \mathrm{~mm}$, and $20 \mathrm{~mm}$, respectively, the cross section of the coil is $20 \mathrm{~mm} \times 3.9 \mathrm{~mm}$ ). This is a benchmark problem solved by other groups and researchers [2], [4]-[6], i.e. the solutions can be checked and compared. 


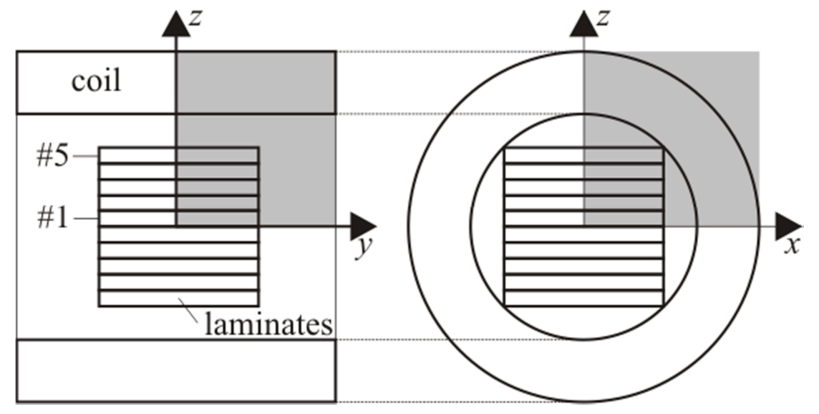

Fig. 1. Geometry of the benchmarked configurations (the shadowed volume is simulated taking symmetry planes into account)

\section{Governing the equations}

The benchmark problem is a coupled problem. The static magnetic field in the air region is coupled through the surface of the laminates to the eddy current field. The general structure of coupled problems is presented in Fig. 2.

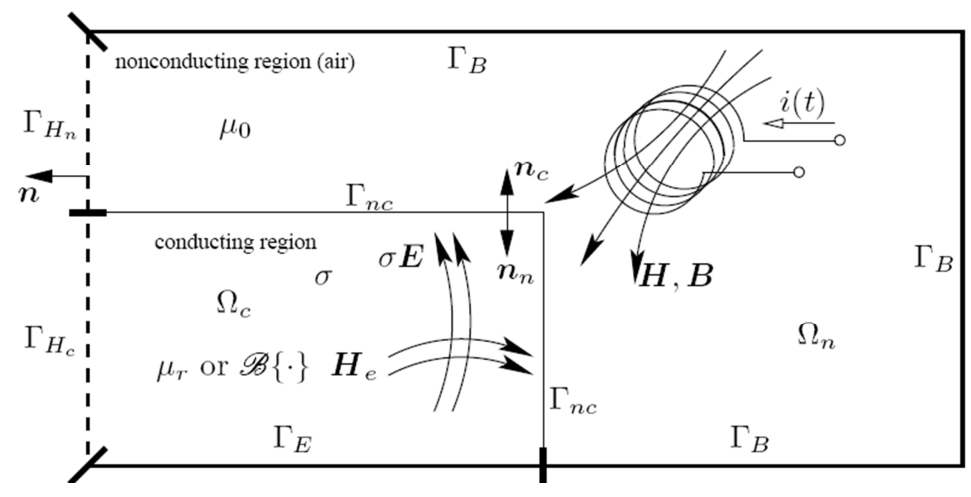

Fig. 2. Structure of an eddy current field problem

The differential form of the Maxwell's equations describing the static magnetic field is as follows [9]-[11]:

$$
\nabla \times \boldsymbol{H}=\boldsymbol{J}_{0}, \nabla \cdot \boldsymbol{B}=0, \boldsymbol{B}=\mu_{0} \boldsymbol{H}, \text { in } \Omega_{n} .
$$

Here $\boldsymbol{H}, \boldsymbol{B}, \boldsymbol{J}_{0}$ and $\mu_{0}$ are the magnetic field intensity, the magnetic flux density, the excitation current density and the vacuum permeability, respectively. Boundary conditions are prescribed on the symmetry plane $\Gamma=\Gamma_{H_{n}} \cup \Gamma_{B}$. The tangential component of the magnetic field intensity and the normal component of the magnetic flux density are vanishing here: $\boldsymbol{H} \times \boldsymbol{n}=\mathbf{0}$ on $\Gamma_{H n}$, and $\boldsymbol{B} \cdot \boldsymbol{n}=0$ on $\Gamma_{B}$. 
The following Maxwell's equations describe the eddy current field inside the laminates [9]-[11]:

$$
\nabla \times \boldsymbol{H}=\boldsymbol{J}=\sigma \boldsymbol{E}, \nabla \times \boldsymbol{E}=-\partial \boldsymbol{B} / \partial t, \nabla \cdot \boldsymbol{B}=0, \boldsymbol{B}=\mu \boldsymbol{H}, \text { in } \Omega_{c} .
$$

Here $\boldsymbol{J}, \boldsymbol{E}$ and $\sigma$ are the eddy current density, the electric field intensity and the conductivity, $\mu$ is the permeability. Only linear characteristics are studied in this paper, i.e. $\mu=\mu_{0} \mu_{r}$ with the relative permeability $\mu_{r}$. Homogeneous boundary conditions are prescribed on the boundary of the eddy current field region $\Gamma=\Gamma_{H_{c}} \cup \Gamma_{E} \cup \Gamma_{n c}$. The tangential component of the magnetic field intensity as well as the electric field intensity is equal to zero on symmetry planes, $\boldsymbol{H} \times \boldsymbol{n}=\mathbf{0}$ on $\Gamma_{H_{c}}, \boldsymbol{E} \times \boldsymbol{n}=\mathbf{0}$ on $\Gamma_{E}$.

In the present problem (Fig. 1), a conducting cube built up from laminates is immersed into the magnetic field of a coil. The tangential component of the magnetic field intensity and the normal component of the magnetic flux density must be continuous on the interface $\Gamma_{n c}$. The normal component of the eddy currents must vanish on the interface.

\subsection{Static magnetic field simulation}

Among many potential formulations [9]-[11], here the magnetic scalar potential $\Phi$ and the magnetic vector potential $\boldsymbol{A}$ are applied to describe static magnetic field in air.

The source current density $\boldsymbol{J}_{0}$ can be represented by the so called impressed field quantity $\boldsymbol{T}_{0}$ by $\boldsymbol{J}_{0}=\nabla \times \boldsymbol{T}_{0}$, because $\nabla \cdot \boldsymbol{J}_{0}=0$. Then, the magnetic field intensity $\boldsymbol{H}$ can be represented by $\boldsymbol{H}=\boldsymbol{T}_{0}-\nabla \Phi$ from the first equation in (1). The following partial differential equation can be obtained from the remained equations in (1):

$$
-\nabla \cdot\left(\mu_{0} \nabla \Phi\right)=-\mu_{0} \nabla \cdot \boldsymbol{T}_{0}, \quad \text { in } \quad \Omega_{n} .
$$

The tangential component of magnetic field intensity can be prescribed by the homogeneous Dirichlet boundary condition $\Phi=0$ on $\Gamma_{H_{n}}$, and the homogeneous Neumann boundary condition $\mu_{0}\left(\boldsymbol{T}_{0}-\nabla \Phi\right) \cdot \boldsymbol{n}=0$ must be prescribed on $\Gamma_{B}$.

Magnetic vector potential $\boldsymbol{A}$ is introduced by the second equation in (1), $\boldsymbol{B}=\nabla \times \boldsymbol{A}$. From the remained equations in (1), the partial differential equation

$$
\nabla \times\left(v_{0} \nabla \times \boldsymbol{A}\right)=\boldsymbol{J}_{0}, \quad \text { in } \quad \Omega_{n}
$$

can be obtained. Here $v_{0}=1 / \mu_{0}$ is called the magnetic reluctivity. The tangential component of magnetic field intensity is prescribed by homogeneous Neumann boundary condition $\left(v_{0} \nabla \times \boldsymbol{A}\right) \times \boldsymbol{n}=\mathbf{0}$ on $\Gamma_{H_{n}}$, and the homogeneous Dirichlet boundary condition $\boldsymbol{n} \times \boldsymbol{A}=\mathbf{0}$ on $\Gamma_{B}$ sets the normal component of magnetic flux density. 


\subsection{Eddy current field simulation}

Two potential functions can be introduced in the eddy current region, either a current vector potential $\boldsymbol{T}$ or a magnetic vector potential $\boldsymbol{A}$. The current vector potential is coupled with a reduced magnetic scalar potential $\Phi$. The magnetic vector potential $\boldsymbol{A}$ is coupled with an electric scalar potential $V$. For details see [9]-[11].

Eddy currents generate solenoid field, i.e. $\nabla \cdot \boldsymbol{J}=0$, from which the current vector potential $\boldsymbol{T}$ can be introduced as $\boldsymbol{J}=\nabla \times \boldsymbol{T}$. The magnetic field intensity can be represented by two unknown potentials as $\boldsymbol{H}=\boldsymbol{T}_{0}+\boldsymbol{T}-\nabla \Phi$ from the first equation in (2). The following partial differential equations can be derived from (2):

$$
\nabla \times(\rho \nabla \times \boldsymbol{T})+\mu(\dot{\boldsymbol{T}}-\nabla \dot{\Phi})=-\mu \dot{\boldsymbol{T}}_{0}, \nabla \cdot(\mu \boldsymbol{T}-\mu \nabla \Phi)=-\nabla \cdot\left(\mu \boldsymbol{T}_{0}\right), \quad \text { in } \quad \Omega_{c} .
$$

Here $\rho=1 / \sigma$ is the resistivity of the material. The homogeneous Dirichlet boundary conditions $\boldsymbol{T} \times \boldsymbol{n}=\mathbf{0}$ and $\Phi=0$ on $\Gamma_{H_{c}}$, furthermore, the homogeneous Neumann boundary conditions $(\rho \nabla \times \boldsymbol{T}) \times \boldsymbol{n}=\mathbf{0}$ and $\mu\left(\boldsymbol{T}_{0}+\boldsymbol{T}-\nabla \Phi\right) \cdot \boldsymbol{n}=0$ on $\Gamma_{E}$ prescribe the boundary conditions of the eddy current field problem.

The following partial differential equations are valid obtained from (2), if the magnetic vector potential is applied:

$$
\nabla \times(v \nabla \times \boldsymbol{A})+\sigma \dot{\boldsymbol{A}}+\sigma \nabla V=\mathbf{0},-\nabla \cdot(\sigma \dot{\boldsymbol{A}}+\sigma \nabla V)=0, \text { in } \Omega_{c}
$$

Here $v=1 / \mu$. The homogeneous Neumann boundary conditions $(v \nabla \times \boldsymbol{A}) \times \boldsymbol{n}=\mathbf{0}$ and $-\sigma(\dot{\boldsymbol{A}}-\nabla V) \cdot \boldsymbol{n}=0$ on $\Gamma_{H_{c}}$, moreover the homogeneous Dirichlet boundary conditions $\boldsymbol{n} \times \boldsymbol{A}=\mathbf{0}$ and $V=0$ on $\Gamma_{E}$ prescribe the boundary conditions of the eddy current field problem.

A third formulation, called the $\boldsymbol{A}, \boldsymbol{T}$-formulation can be developed by applying the potentials $\boldsymbol{A}$ and $\boldsymbol{T}$ according to $\boldsymbol{B}=\nabla \times \boldsymbol{A}$ and $\boldsymbol{J}=\nabla \times \boldsymbol{T}$ [12]:

$$
\nabla \times(v \nabla \times \boldsymbol{A})-\nabla \times \boldsymbol{T}=\mathbf{0}, \nabla \times(\rho \nabla \times \boldsymbol{T})+\nabla \times \dot{\boldsymbol{A}}=\mathbf{0}, \quad \text { in } \quad \Omega_{c} .
$$

The homogeneous boundary conditions $(\nu \nabla \times \boldsymbol{A}) \times \boldsymbol{n}=\mathbf{0}$ and $\boldsymbol{T} \times \boldsymbol{n}=\mathbf{0}$ on $\Gamma_{H_{c}}$, moreover $\boldsymbol{n} \times \boldsymbol{A}=\mathbf{0}$ and $(\rho \nabla \times \boldsymbol{T}) \times \boldsymbol{n}=\mathbf{0}$ on $\Gamma_{E}$ prescribe the boundary conditions of the eddy current field problem.

The potential formulations are solved numerically by the finite element method. By applying the Galerkin's weighted residual method to the above mentioned formulations, symmetric equations can be obtained. The potentials are approximated by nodal and edge finite elements, i.e. the gauged and the ungauged versions of the formulations have been tested. It is noted that the equations have been solved by time harmonic analysis. The presented issues have been realized in the frame of COMSOL Multiphysics 3.5.a [14]. For details in the numerical implementation, refer to [9]-[13]. 


\section{The reference model}

The reference solution can be obtained by the $T, \Phi-\Phi$-formulation in a trivial way. Either nodal or edge element based approximation can be used. Laminations are modeled exactly by prescribing $\boldsymbol{T} \times \boldsymbol{n}=\mathbf{0}$ on the boundaries between the sheets. This is Dirichlet boundary condition to prescribe $\boldsymbol{J} \cdot \boldsymbol{n}=0$. Of course, the finite element mesh is fine and it results in a large number of unknowns.

Unfortunately, this formulation cannot be applied in real life problems, because of the extreme amount of computational costs. However, it is useful as a reference solution in this simple benchmark problem.

\section{The bulk model}

The bulk model takes the laminar nature of eddy currents into account by assuming an anisotropic conductivity with its value negligible in the direction perpendicular to the laminates (this is the $z$ direction, see Fig. 1), i.e. the conductivity tensor is as follows:

$$
\boldsymbol{\sigma}=\left[\begin{array}{ccc}
\sigma_{x} & 0 & 0 \\
0 & \sigma_{y} & 0 \\
0 & 0 & \sigma_{z}
\end{array}\right] \text {, and } \boldsymbol{\rho}=\left[\begin{array}{ccc}
1 / \sigma_{x} & 0 & 0 \\
0 & 1 / \sigma_{y} & 0 \\
0 & 0 & 1 / \sigma_{z}
\end{array}\right]
$$

with the components $\sigma_{\mathbf{x}}=\sigma_{y}=\sigma$ and $\sigma_{\mathbf{z}}=0$ or very low. In (5)-(7) $\sigma$ and $\rho$ are replaced by the tensors in (8). Now, it is easy to understand that $\sigma_{\mathbf{z}}=0$ can only be approximated if formulations containing the potential $\boldsymbol{T}$ are applied (see (5) and (7)). Potential $\boldsymbol{A}$ in (6) has a natural advantage that $\sigma_{\mathbf{z}}=0$ can be set exactly.

Three potential formulations have been tested, the T, $\Phi$ - $\Phi$-formulation, the $\mathbf{A}, \mathrm{V}-\mathbf{A}-$ formulation and the $\mathbf{A}, \mathbf{T}-\mathbf{A}$-formulation approximated by nodal and edge finite elements. The observations are described in this section.

\subsection{The $\boldsymbol{T}, \Phi-\Phi$-formulation}

The equations (3) and (5) set up the formulation. The value of $\sigma_{\mathbf{z}}$ cannot be set to zero. After some trials, it is found that $\sigma_{\mathbf{z}}=\sigma / c$ with $c=1000$ results in an adequate approximation. However, the number of iterations to find the solution by iterative solvers is increasing by increasing the value of $c$, i.e. the computation time is increasing.

It is an experiment that finer mesh is necessary to obtain applicable solution than in the case of the $\boldsymbol{A}, V$ - $\boldsymbol{A}$-formulation.

\subsection{The $\boldsymbol{A}, \boldsymbol{V}$-A-formulation}

The formulation is based on (4) and (6). In this formulation $\sigma_{\mathbf{z}}=0$ can be prescribed exactly. 
It is found that the nodal approximation as well as the solution by edge elements results in similar performance.

\subsection{The $\boldsymbol{A}, \boldsymbol{T}$ - $\boldsymbol{A}$-formulation}

Equation (4) is coupled to (7) in this formulation. The value of $\sigma_{\mathbf{z}}$ cannot be set to zero. The performance of this formulation is similar to that of the $\mathbf{T}, \Phi-\Phi$-formulation.

Fig. 3 shows the real part of the magnetic flux density vectors within the cube. After comparing the results of the reference model and the three bulk models, it can be concluded that the magnetic field distribution yielded by bulk models closely approximates the field obtained by the reference model.

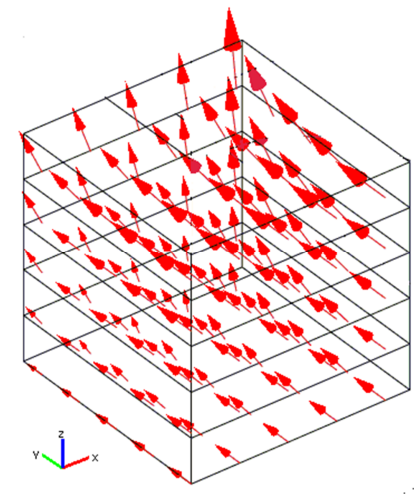

Fig. 3. The real part of the magnetic flux density within the cube

The bulk models represent the eddy current field distribution in a weak sense as it is presented in Fig. 4. The maximum value of the eddy current field is about ten times higher by the bulk models than the reference one.
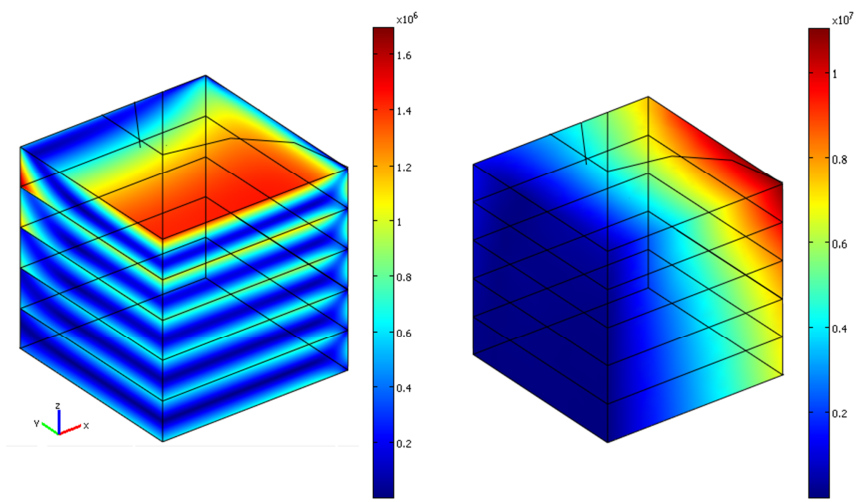

Fig. 4. Comparison of the real part of the eddy current density on the surface of the modeled cube simulated by the reference model and the bulk models 
It is noted that the preferred technique to solve the bulk model is the A,V-Aformulation.

\section{The model of individual laminates}

The magnetic field distribution calculated by the bulk models can be used to obtain the boundary condition of the individual laminates [5], [6]. The tangential component of the magnetic field intensity prescribed on the boundary of a laminate determines the electromagnetic field within the sheet uniquely. There is another way to get unique solution. The normal component of the magnetic flux density on the boundary must be prescribed together with the specification of the normal component of the current density. The latter is zero. These boundary conditions can be set by the potential formulations as it is presented next.

\subsection{The $\boldsymbol{T}$, $\Phi$-formulation}

The tangential component of the magnetic field intensity on the boundary of each laminate can be prescribed easily if the T, $\Phi-\Phi$-formulation is applied to solve the bulk model. The boundary conditions are as follows:

$$
\boldsymbol{T} \times \boldsymbol{n}=\left(\boldsymbol{T}_{b}-\nabla \Phi_{b}\right) \times \boldsymbol{n}, \text { and } \Phi=0 .
$$

Here, the subscript ' $b$ ' refers to the potentials obtained from the bulk model.

\subsection{The $A, V$-formulation}

The normal component of the magnetic flux density on the boundary of each laminate can be prescribed easily if the $\boldsymbol{A}, \boldsymbol{V}$ - $\boldsymbol{A}$-formulation is applied to solve the bulk model. The following boundary conditions must be specified:

$$
\boldsymbol{A} \times \boldsymbol{n}=\boldsymbol{A}_{b} \times \boldsymbol{n} \text {, and } V \text { is free. }
$$

\subsection{The A, $\boldsymbol{T}$-formulation}

The normal component of the magnetic flux density on the boundary of each laminate is prescribed in the same way as in the aforementioned section. Moreover, the normal component of the eddy current density can be set to zero by prescribing the tangential component of the current vector potential to zero. Finally, the following boundary conditions are used:

$$
\boldsymbol{A} \times \boldsymbol{n}=\boldsymbol{A}_{b} \times \boldsymbol{n}, \text { and } \boldsymbol{T} \times \boldsymbol{n}=\mathbf{0} .
$$

The component $\boldsymbol{A}_{b}$ can be obtained either from the $\boldsymbol{A}, V$ - $\boldsymbol{A}$-formulation or the $\boldsymbol{A}, \boldsymbol{T}-\boldsymbol{A}$ formulation. 
The finite element mesh of individual models is very simple; the triangular mesh of the sheet boundary $x-y$ is extruded in the $z$ direction. It is found that three layers of the mesh results in appropriate solution. Of course, it is depending on the skin depth.

Fig. 5 and Fig. 6 compare the real and imaginary part of the magnetic field intensity and the eddy current density, respectively, simulated by all the above presented methods. The reference solution and the results of bulk models and individual models are shown. The line where the comparison is performed is along $x=2 \mathrm{~mm}, y=2 \mathrm{~mm}$, and $z=0 \ldots 5 \mathrm{~mm}$ (see Fig. 1). The boundary of the sheets is represented by dotted lines. All the three individual models showed in this session yield similar results.

It can be concluded that the individual analysis of the laminates gives very attractive results for the magnetic field and the eddy current field, too.
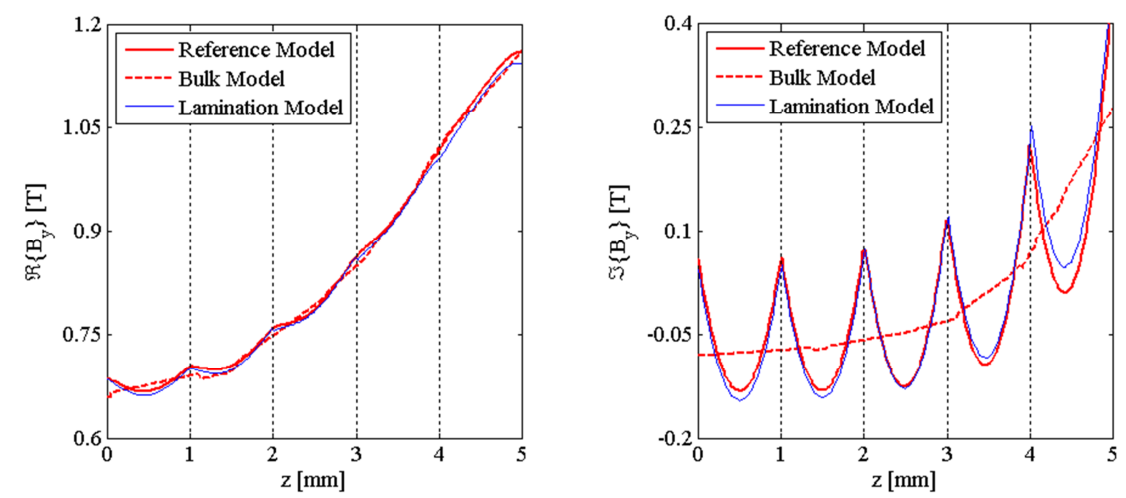

Fig. 5. Comparison of the magnetic flux density
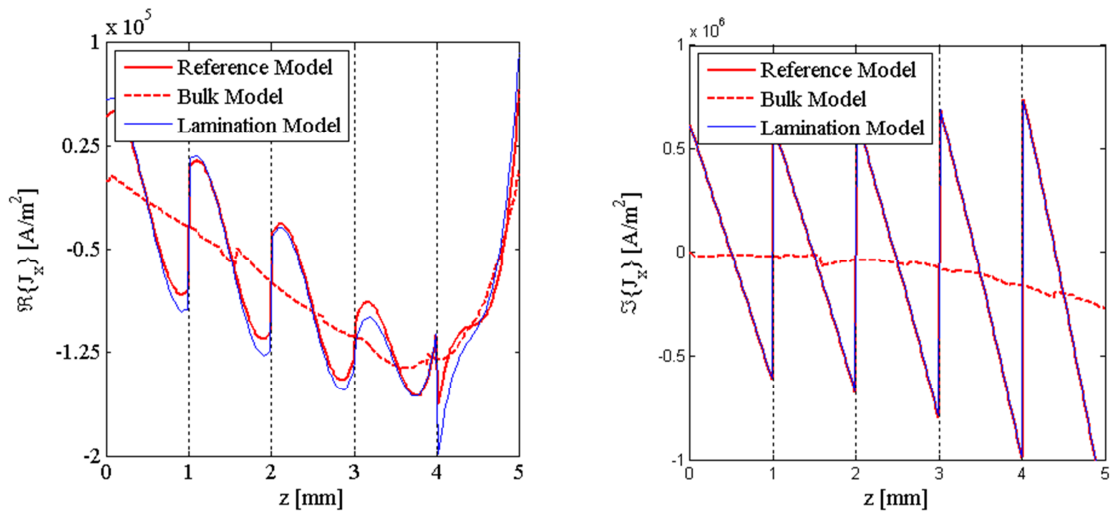

Fig. 6. Comparison of the eddy current density

Fig. 7 shows the real part of the magnetic flux density vector within the laminates and the eddy current density on the boundary of the individual sheets. These results show very good agreement with the reference model. 

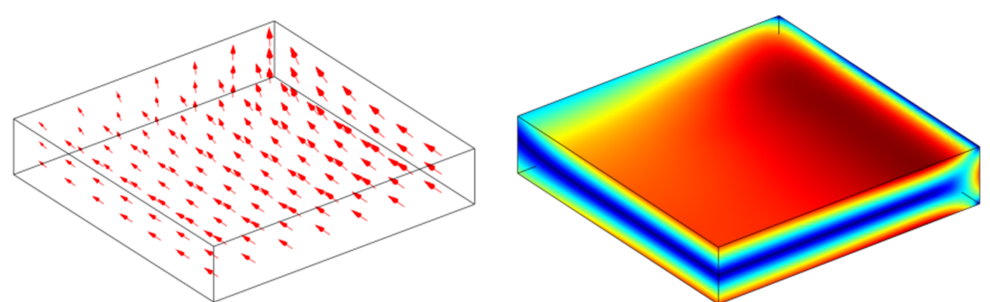

a) Laminate \#1
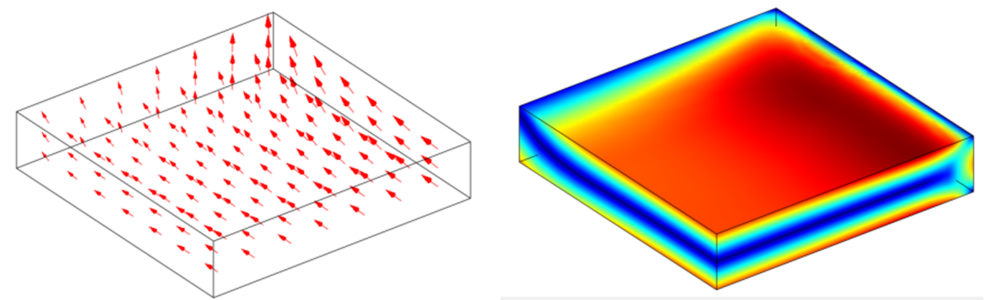

b) Laminate \#2
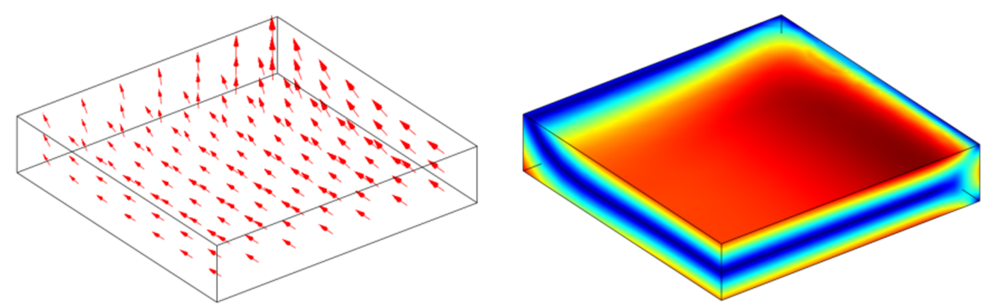

c) Laminate \#3
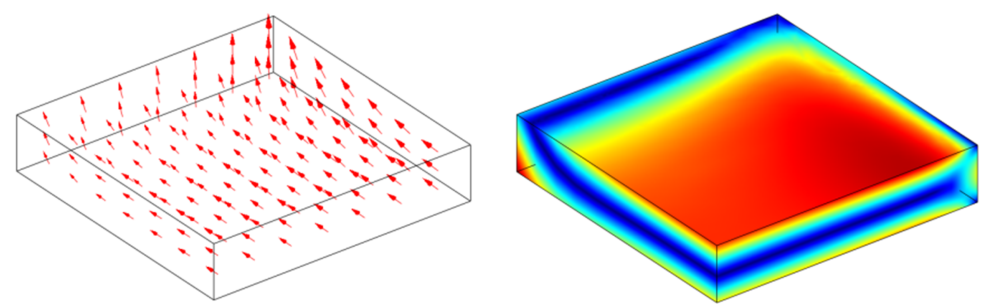

d) Laminate \#4

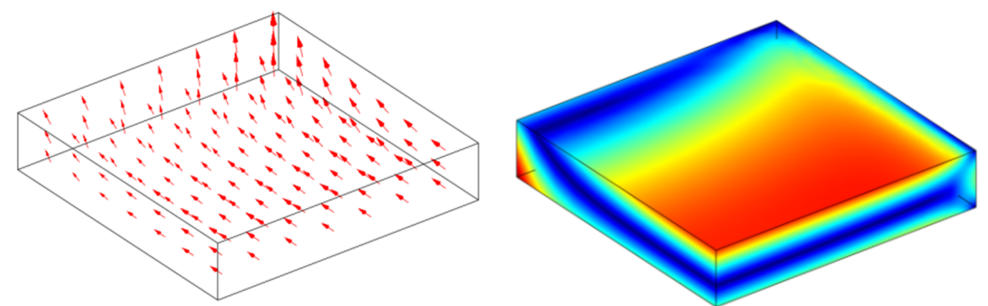

e) Laminate \#5

Fig. 7. The magnetic flux density and eddy current density within the laminates 
The eddy current loss has been calculated by the aforementioned models by

$$
P_{e}=\int_{\Omega_{c}} \frac{|\boldsymbol{J}|^{2}}{\sigma} d \Omega
$$

Here, $\boldsymbol{J}$ is the complex amplitude of the eddy current density. Table I shows the comparison between the eddy current field losses calculated by the reference model and the three mentioned formulations of the individual laminates. The relative error is also presented. Numbers $\# 1$...\#5 denote the laminates in Fig. 1. As it can be stated, all the three formulations yield almost the same results.

\section{Table I}

Comparison between the different formulations, power loss (dimensions are in $\mathrm{mW}$ )

\begin{tabular}{|l|l|l|l|l|l|l|l|}
\cline { 2 - 8 } \multicolumn{1}{c|}{} & \multicolumn{1}{c|}{ Reference } & \multicolumn{2}{c|}{$\boldsymbol{A}, \boldsymbol{V}$-formula } & \multicolumn{2}{c|}{$\boldsymbol{A}, \boldsymbol{T}$-formula } & \multicolumn{2}{c|}{$\boldsymbol{T}, \Phi$-formula } \\
\hline$\# 1$ & 0.2751 & 0.2726 & $-0.9 \%$ & 0.2726 & $-0.9 \%$ & 0.2666 & $-3.1 \%$ \\
$\# 2$ & 0.3230 & 0.3206 & $-0.7 \%$ & 0.3205 & $-0.7 \%$ & 0.3283 & $+1.6 \%$ \\
$\# 3$ & 0.4398 & 0.4373 & $-0.6 \%$ & 0.4372 & $-0.6 \%$ & 0.4403 & $+0.1 \%$ \\
$\# 4$ & 0.6682 & 0.6573 & $-1.6 \%$ & 0.6572 & $-1.6 \%$ & 0.6719 & $+0.6 \%$ \\
$\# 5$ & 1.0128 & 0.9832 & $-2.9 \%$ & 0.9829 & $-2.9 \%$ & 1.1249 & $+11 \%$ \\
\hline Sum & 2.7189 & 2.6710 & $-1.8 \%$ & 2.6704 & $-1.8 \%$ & 2.8320 & $+4.2 \%$ \\
\hline
\end{tabular}

It is difficult to choose the best formulation for simulating the individual laminates, because the performance of the three techniques is very similar, either applying nodal or edge finite element approximation.

All in all, the $\boldsymbol{A}, V$ - $\boldsymbol{A}$-formulation for the bulk model with the $\boldsymbol{A}, V$-formulation or the $\boldsymbol{A}, \boldsymbol{T}$-formulation for the individual model seems to be the best choice.

These formulations will be used in the next study to investigate asynchronous machines [15]-[17].

\section{Conclusion}

This study has shown that the eddy current field within laminates can be computed efficiently by the investigated two-step method. The bulk model presumes anisotropic conductivity but laminates are not modeled. The boundary conditions of individual models are yielded by the bulk model. The sheets can be simulated by parallel computation, because laminate fields are independent from other. The evidence from this work suggests that the $\boldsymbol{A}, V$ - $\boldsymbol{A}$-formulation for the bulk model and the $\boldsymbol{A}, V$ formulation or the $\boldsymbol{A}, \boldsymbol{T}$-formulation for the individual model seems to be the best choice.

The current research has only examined linear constitutive relationship between the magnetic field intensity and the magnetic flux density. Further work needs to be done to insert hysteresis models. A future study investigating asynchronous machines is also planned. Considerably more work is needed to be done to determine hysteresis loss, eddy current loss and excess loss in a real-life machine. 


\section{Acknowledgements}

This work has been supported by the ÚNKP-17-4 New National Excellence Program of the Ministry of Human Capacities.

\section{References}

[1] Handgruber P. Advanced eddy current and hysteresis loss models for steel laminations of rotating electrical machines, $P h D$ Theses, University of Technology of Graz, Graz, 2015.

[2] Hollaus K. Numerical simulation of eddy currents and the associated losses in laminated ferromagnetic materials by the method of finite elements, $P h D$ Theses, University of Technology of Graz, Graz, 2001.

[3] Silva V. C., Meunier G., Foggia A. A 3-D finite element computation of eddy currents and losses in laminated iron cores allowing for electric and magnetic anisotropy, IEEE Trans. Magn. Vol. 31, 1995, pp. 2139-2141.

[4] Sebestyén I., Gyimóthy Sz., Pávó J., Bíró O. Calculation of losses in laminated ferromagnetic materials, IEEE Trans. Magn. Vol. 40, 2004, pp. 924-927.

[5] Bíró O., Preis K., Ticar I. A FEM method for eddy current analysis in laminated media, Proc. of the 11th International Symposium on Electromagnetic Fields in Electrical Engineering, Maribor, Slovenia, 18-20 September, 2003, pp. 9-14.

[6] Bíró O., Preis K., Ticar I. A FEM method for eddy current analysis in laminated media, COMPEL, Vol. 24, No. 1, 2006, pp. 241-248.

[7] Jack A. G., Mecrow B. C. Calculation of three-dimensional electromagnetic fields involving laminar eddy currents, IEE Proceedings, Vol. 134 Pt. A, 1987, pp. 663-671.

[8] Emad D. Magnetodynamic vector hysteresis models for steel laminations of rotating electrical machines, PhD Theses, Helsinki University of Technology, Helsinki, 2008.

[9] Kuczmann M. Nodal and vector finite elements in static and eddy current field problems, Pollack Periodica, Vol. 3, No. 2, 2008, pp. 85-96.

[10] Kuczmann M., Iványi A. The finite element method in magnetics, Budapest, Academic Press, 2008.

[11] Bíró O., Richter K. R. CAD in electromagnetism, Advances in Electronics and Electron Physics, Vol. 82, 1991, pp. 1-96.

[12] Albertz D., Henneberger G. Calculation of 3D eddy current fields using both electric and magnetic vector potential in conducting region, IEEE Trans. Magn. Vol. 34, No. 5, 1998, pp. 2644-2647.

[13] Benes S., Kruis J. Approximation methods for post-processing of large data from the finite element analysis, Pollack Periodica, Vol. 11, No. 3, 2016, pp. 165-176.

[14] www.comsol.com, (last visited 15 October 2017).

[15] Kapeller H., Dvorak D., Gragger V. J., Müllner F., Neudorfer H. Modeling of iron losses in an induction machine based on magnetic equivalent circuit in Modelica, Proceedings of the 2017 IEEE International Electric Machines and Drives Conference, 21-24 May 2017, Miami FL, USA, pp. 1-8.

[16] Lehikonien A., Ikaheimo J., Arkkio A., Belahcen A. Domain decomposition approach for efficient time-domain finite-element computation of winding losses in electrical machines, IEEE Trans. Magn. Vol. 53, No. 5, 2017, Paper No. 7400609.

[17] Dems M., Komeza K. The influence of electrical sheet on the core losses at no-load and full-load of small power induction motors, IEEE Trans. Ind. El. Vol. 64, No. 3, 2017, pp. 2433-2442 